\title{
Effect of Beetroot Juice Supplementation on Mood, Perceived Exertion, and Performance During a 30-Second Wingate Test
}

\author{
Pablo Jodra, Raúl Domínguez, Antonio J. Sánchez-Oliver, Pablo Veiga-Herreros, \\ and Stephen J. Bailey
}

\begin{abstract}
Purpose: Dietary supplementation with inorganic nitrate $\left(\mathrm{NO}_{3}{ }^{-}\right)$can enhance high-intensity exercise performance by improving skeletal muscle contractility and metabolism, but the extent to which this might be linked to altered psychophysiological processes is presently unclear. The purpose of this study was to assess the effects of $\mathrm{NO}_{3}{ }^{-}$-rich beetroot juice (BJ) supplementation on profile of mood states, ratings of perceived exertion (RPE), and performance in a 30-second Wingate cycle test. Methods: In a double-blind, randomized, cross-over study, 15 subjects completed 2 laboratory sessions after ingesting $\mathrm{NO}_{3}{ }^{-}$-rich or $\mathrm{NO}_{3}{ }^{-}$-depleted (placebo) BJ. Participants initially completed the profile of mood states questionnaire. Subsequently, participants completed a warm-up followed by a 30-second all-out Wingate cycling test. After the Wingate test, participants immediately indicated the RPE of their leg muscles (RPE $E_{\text {muscular }}$ ), cardiovascular system (RPE $\left.\mathrm{cardio}\right)$, and general RPE (RPE general $)$. Results: Compared with the placebo condition, supplementation with BJ increased peak power output $\left(W_{\text {peak }}\right)$ $\left(+4.4 \%, 11.5[0.7]\right.$ vs $\left.11.1[1.0] \mathrm{W} \cdot \mathrm{kg}^{-1} ; P=.039\right)$ and lowered the time taken to reach $W_{\text {peak }}(7.3[0.9] \mathrm{vs} 8.7[1.5] \mathrm{s} ; P=.002)$ during the Wingate test. The profile of mood states score linked to tension was increased prior to the Wingate test (4.8 [3.0] vs 3.4 [2.4]; $P=.040)$, and $\mathrm{RPE}_{\text {muscular }}$ was lowered immediately following the Wingate test (17.7 [1.6] vs 18.3 [1.0]; $\left.P=.031\right)$, after BJ compared with placebo ingestion. Conclusions: Acute BJ supplementation improved pre-exercise tension, 30-second Wingate test performance, and lowered postexercise RPE $E_{\text {muscular }}$.
\end{abstract}

Keywords: dietary supplement, ergogenic aid, high-intensity exercise, nitrate, mood states

In high-performance sports, even a small increment in performance can have a large impact on competition outcome. With this in mind, many athletes attempt to boost their performance through the consumption of purported nutritional ergogenic aids. However, although many commercially available supplements claim to improve sports performance, such claims are not always supported by a firm foundation of robust scientific evidence. To overcome this ambiguity and to provide evidence-based recommendations for dietary supplementation to enhance sports performance, the International Olympic Committee has recently published a classification for nutritional supplements based on the scientific evidence to support their ergogenic efficacy. ${ }^{1}$ One dietary supplement classified as having a high level of scientific evidence to support an ergogenic effect was inorganic nitrate $\left(\mathrm{NO}_{3}{ }^{-}\right)$.

The ergogenic effects of $\mathrm{NO}_{3}{ }^{-}$supplementation, which is 1 tr.mically administered in the form of $\mathrm{NO}_{3}{ }^{-}$-rich beetroot juice I), are attributed to its stepwise reduction to nitrite and, subsequently, nitric oxide (NO) as $\mathrm{NO}_{3}{ }^{-}$is considered biologically inert. ${ }^{2}$ After $\mathrm{NO}_{3}{ }^{-}$supplementation, the increase in plasma [nitrite] serves as a circulating substrate for $\mathrm{O}_{2}$-independent $\mathrm{NO}$ generation, with the reduction of nitrite to $\mathrm{NO}$ enhanced in conditions of hypoxia and acidosis. ${ }^{2}$ Some of the physiological processes that have been reported to be enhanced by increased NO exposure,

Jodra and Veiga-Herreros are with the College of Health Sciences, Alfonso X El Sabio University, Madrid, Spain. Domínguez is with the Faculty of Health Sciences, Isabel I University, Burgos, Spain. Sánchez-Oliver is with the Faculty of Sports Sciences, Pablo de Olavide University, Seville, Spain; and the Human Motricity and Sports Performance Area, University of Seville, Seville, Spain. Bailey is with the School of Sport, Exercise and Health Sciences, Loughborough University, Loughborough, United Kingdom. Domínguez (raul.dominguez@ui1.es) is corre- which might underpin the ergogenic effects of $\mathrm{NO}_{3}{ }^{-}$supplementation, include improvements in muscle vasodilation and blood flow, ${ }^{3}$ metabolic responses ${ }^{4}$ and contractile force. ${ }^{5}$ Moreover, it is well documented that type II, fast-twitch skeletal muscle is more hypoxic ${ }^{3}$ and acidic $^{6}$ compared with type I, slow-twitch muscle during contractions, and that type II muscle is heavily recruited during high-intensity intermittent and all-out sprint exercise. ${ }^{7}$ Therefore, the potential for an ergogenic effect following dietary $\mathrm{NO}_{3}{ }^{-}$supplementation might be greatest during short-duration, high-intensity exercise, as supported by improved single sprint and/or repeated sprint/high-intensity intermittent exercise performance following $\mathrm{NO}_{3}{ }^{-}$supplementation. ${ }^{8}$

In addition to physiological factors within the skeletal muscle, it is recognized that psychological factors, such as mood and ratings of perceived exertion (RPE), can play a role in determining exercise performance. ${ }^{9}$ There is some evidence to suggest that ergogenic supplements, when consumed in large amounts, may raise levels of subjective tension, ${ }^{10}$ which is considered the internal sensation of preparation to react immediately and with sufficient intensity to a demanding task. ${ }^{11}$ However, the general consensus is that psychophysiological activation leads to improved performance up to a certain tension threshold, with diminished performance manifesting above this critical threshold. ${ }^{12}$ In addition, subjective RPE, which characterizes the combination of feelings related to the execution of a physical exercise task, and has been considered to reflect the integrated response of feedback from central, peripheral, and metabolic factors, ${ }^{13}$ has been reported to exhibit a positive correlation with objective physiological indicators. ${ }^{7}$ However, the effect of $\mathrm{NO}_{3}{ }^{-}$supplementation on RPE is equivocal, ${ }^{14,15}$ and no investigation has partitioned the effect of $\mathrm{NO}_{3}{ }^{-}$supplementation on general, cardiovascular and muscular RPE. Moreover, the effect of $\mathrm{NO}_{3}{ }^{-}$supplementation on other 
psychological components, such as mood, is undefined, despite some evidence that $\mathrm{NO}_{3}{ }^{-}$intake can improve brain blood flow, ${ }^{16}$ which is an important determinant of RPE and mood state profile. ${ }^{17}$

The purpose of this study was to assess the effects of $\mathrm{NO}_{3}{ }^{-}$supplementation on RPE, mood profile, and performance in a 30-second Wingate cycle test in resistance-trained males. Resistance-trained participants were selected on the basis that resistance training elicits greater skeletal muscle hypertrophy in type II compared with type I muscle fibers such that a greater portion of muscle volume is likely composed of type II muscle in resistance-trained participants. ${ }^{18}$ Therefore, resistance-trained participants might be particularly well placed to exhibit improved sprint performance following $\mathrm{NO}_{3}{ }^{-}$supplementation. It was hypothesized that, compared with a $\mathrm{NO}_{3}{ }^{-}$-depleted $\mathrm{BJ}$ placebo, a $\mathrm{NO}_{3}{ }^{-}$-rich BJ would improve mood, lower RPE, and enhance 30-second Wingate cycle test performance.

\section{Materials and Methods}

\section{Study Participants}

Fifteen resistance-trained, male undergraduate students participated in this study (see Table 1 for the participant characteristics). Participation was voluntary after prospective participants met the following inclusion criteria, which were established in a preliminary prescreening session: (1) having completed at least 3 sessions per week of strength training within the past 18 months; (2) a bench press 1-repetition maximum greater than body mass and full-squat 1-repetition maximum at least 1.5 times greater than body mass; (3) no nutritional supplements had been consumed for at least 3 months before the study onset; (4) nonsmoker; (5) no cardiovascular, respiratory, metabolic, neurological, or orthopedic disorders that could interfere with cycle ergometer performance; (6) not a full-time professional athlete; and (7) experience with the Wingate test, having performed at least one test in the 3 months preceding the study commencement. Moreover, the investigators informed the participants of the study goals and test protocols, including the dietary requirements to be followed and the avoidance of other dietary supplements, during this prescreening session. After participants met the inclusion criteria and agreed to participate, they provided their written informed consent to participate in the study. The study protocol was approved by the ethics committee of the Universidad Alfonso X El Sabio (Madrid, Spain).

\section{Study Design}

The study adopted a randomized, double-blind, cross-over experimental design. Each participant completed 2 test sessions separated by 72 hours in an exercise physiology laboratory at the same time of day $( \pm 0.5 \mathrm{~h})$. Subjects were instructed to refrain from any type of physical exercise for 72 hours before the first session until the end

Table 1 Participant Characteristics

\begin{tabular}{lc}
\hline Variable & Value \\
\hline Age, $\mathrm{y}$ & $23(2)$ \\
Height, $\mathrm{m}$ & $1.78(0.06)$ \\
Weight, $\mathrm{kg}$ & $75.6(8.9)$ \\
Body mass index, $\mathrm{kg} / \mathrm{m}^{2}$ & $23.9(2.1)$ \\
\hline
\end{tabular}

Note: Data are expressed as mean (SD). of the study. All subjects were given strict guidelines to ensure their diet comprised a similar macronutrient composition (60\% carbohydrates, $30 \%$ lipids, and $10 \%$ proteins) during the investigation. Participants were instructed to record their food intake for 48 hours prior to the first supplementation trial and to reproduce this prior to the second supplementation trial. Upon arrival at the laboratory for each session, participants ingested BJ or placebo. In the first experimental session, 8 subjects ingested BJ supplementation and 7 subjects ingested placebo. One hundred and fifty minutes after ingesting the supplement, participants completed the profile of mood states (POMS) questionnaire. Participants then completed a 30-second Wingate test on a cycle ergometer after a warm-up. Immediately after the test, participants graded their exertion using the RPE scale (see Figure 1).

BJ Supplementation. Participants arrived at the laboratory 3 hours before initiating the Wingate test. Upon arrival, participants consumed a 70-mL BJ supplement that was either enriched in $\mathrm{NO}_{3}{ }^{-}$ $\left(\sim 6.4 \mathrm{mmol}\right.$ of $\left.\mathrm{NO}_{3}{ }^{-}\right)$or depleted in $\mathrm{NO}_{3}{ }^{-}\left(0.04 \mathrm{mmol}\right.$ of $\left.\mathrm{NO}_{3}{ }^{-}\right)$as placebo (Beet It; James White Drinks Ltd, Ipswich, UK). The timing of BJ ingestion was based on the recommendation of ingesting the supplement 2.5 to 3 hours before starting an exercise effort to coincide with peak plasma $\left[\mathrm{NO}_{2}^{-}\right]$. To avoid a potential confounding influence from habitual dietary $\mathrm{NO}_{3}{ }^{-}$intake, subjects were given a list of foods high in $\mathrm{NO}_{3}{ }^{-}$to avoid for 48 hours before each session. Furthermore, to control for a possible ergogenic effect of caffeine ingestion on test performance, the intake of caffeine was also restricted 24 hours before the study start, and the subjects were provided with a list of foodstuffs rich in caffeine to avoid. Finally, subjects were instructed to avoid brushing their teeth on the morning of testing and the use of antibacterial mouthwash, which would alter the oral microbiota and interfere with $\mathrm{NO}_{3}{ }^{-}$reduction, from 1 week prior to the first laboratory visit and for the duration of the study.

Profile of Mood States. To assess the participants' mood, the POMS questionnaire was used in its original reduced version, which has been translated into Spanish and validated by Fuentes et al. ${ }^{19}$ Participants graded a set of 29 adjectives related to mood on a Likert scale from 0 (not at all) to 4 (extremely) in reply to the question "How do you feel at this moment?" to assess 6 dimensions: tension (T), depression (D), anger (A), vigor (V), fatigue (F), and confusion $(\mathrm{C})$.

Wingate Test. The Wingate test was completed on a cycle ergometer (Ergomedic 828E; Monark Exercise, Vansbro, Sweden). Prior to completing the Wingate test, participants performed a 5-minute warm-up at a self-selected submaximal cycling workload. After 1 minute of passive rest, subjects subsequently completed a specific warm-up comprising 3 minutes of cycle exercise at $120 \mathrm{~W}$ (60 rpm), with a maximum 5-second sprint completed at the end of each minute. After 2 minutes of rest, the Wingate test was completed.

The Wingate test consisted of 30-second maximal cycling, commenced with the pedals stationary, with the resistance on the flywheel set to $7.5 \%$ of the participant's body mass. Participants were instructed to (1) commence the first pedal stroke with the dominant leg, (2) reach the maximum rpm in the shortest time possible, and (3) provide a maximal effort to maintain this pedaling speed until the end of the test. Power output was recorded during each second of the test. The following variables were subsequently calculated: peak power $\left(W_{\text {peak }}\right)$, the time to reach $W_{\text {peak }}$ (time-to$\left.W_{\text {peak }}\right)$, mean $W$ for the test duration $\left(W_{\text {mean }}\right)$, and minimum power $\left(W_{\min }\right)$, taken as the lowest $W$ recorded during the last 10 seconds of the test. 


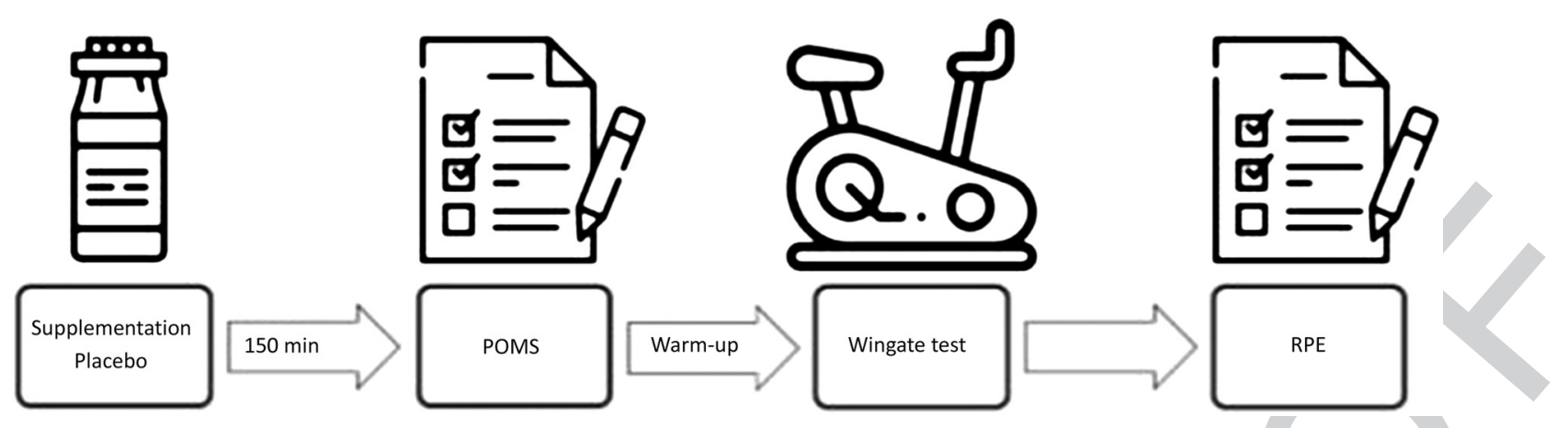

Figure 1 - Flow diagram showing the different stages of each experimental session. POMS indicates profile of mood states; RPE, rating of perceived exertion.

tings of Perceived Exertion. As soon as participants had npleted the Wingate test, they were presented with the 6 to 20 RPE scale. Participants were then asked to indicate the RPE related to their leg muscles ( $\mathrm{RPE}_{\text {muscular }}$ ), cardiovascular system $\left(\mathrm{RPE}_{\text {cardio }}\right)$, and general overall RPE (RPE $\left.\mathrm{general}\right)$.

\section{Statistical Analysis}

All data were initially tested for normal distribution using ShapiroWilk tests. Subsequently, Student $t$ tests were used to compare the outcome variables between the 2 experimental conditions (placebo and BJ). The Wilcoxon test was used for data that were not normally distributed ( $\mathrm{RPE}_{\text {muscular }}$ and $\left.\mathrm{RPE}_{\text {general }}\right)$. Effect size (ES) was calculated using Cohen $d$ with values of $<0.2,0.5$ to 0.8 , and $>0.8$ reflective of trivial, moderate, and large ESs, respectively. All data are reported as mean (SD). Statistical significance was set at $P<.05$. Statistical tests were performed using SPSS (version 18.0; SPSS, Chicago, IL).

\section{Results}

The $W_{\text {peak }}$, time-to- $W_{\text {peak }}, W_{\text {mean }}$, and $W_{\text {min }}$ variables are illustrated for a representative individual in Figure 2 and presented as group mean values in Figure 3 . The $W_{\text {peak }}$ was higher $(+4.4 \%, 11.5$ [0.7] vs $11.1[1.0] \mathrm{W} / \mathrm{kg} ; t=-2.280, \mathrm{ES}=0.48, P=.039$; Figure 3 ), and time-to- $W_{\text {peak }}$ was lower $(7.3[0.9]$ vs 8.7 [1.5] s; $t=3.898$, $\mathrm{ES}=1.17, P=.002$; Figure 2) after BJ compared with placebo

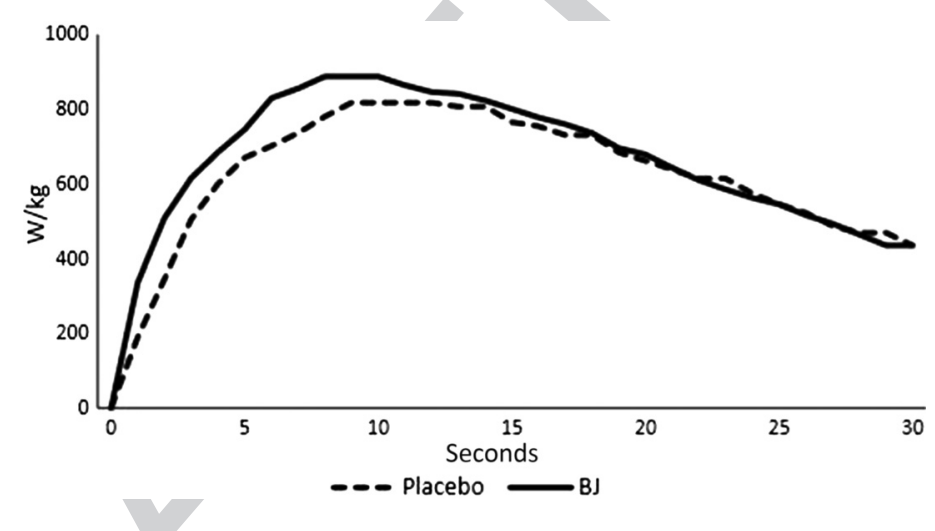

Figure 2 - Power output (W/kg) profile during the 30-second Wingate test for a representative subject in the placebo and BJ conditions. $\mathrm{BJ}$ indicates beetroot juice. ingestion. There were no differences in $W_{\text {mean }}$ and $W_{\min }$ between the $\mathrm{BJ}$ and placebo conditions ( $W_{\text {mean }}: 8.6[0.6]$ vs $8.5[0.8] \mathrm{W} / \mathrm{kg}$; $t=-1.379, \mathrm{ES}=0.19, P=.104$ and $W_{\min }: 6.2[0.8]$ vs $6.0[0.9] \mathrm{W} / \mathrm{kg}$; $t=-1.064, \mathrm{ES}=0.24, P=.305$; Figure 3 ).

For the different POMS dimensions, a higher tension score was reported in the BJ condition (4.80 [2.98]) compared with the placebo condition (3.40 [2.38]; $\mathrm{ES}=0.53, P=.040)$. No significant differences between the 2 experimental conditions were detected in any of the other POMS dimensions (Table 2).

Immediately following the Wingate test, $\mathrm{RPE}_{\text {muscular }}$ was lower in the BJ compared with the placebo condition (17.7 [1.6] vs $18.3[1.0] ; z=-2.157, \mathrm{ES}=0.47, P=.031)$. There were no differences in $\mathrm{RPE}_{\text {cardio }}$ and $\mathrm{RPE}_{\text {general }}$ between the $\mathrm{BJ}$ and placebo conditions $\left(\mathrm{RPE}_{\mathrm{cardio}}=17.4[1.6]\right.$ vs 17.7 [1.63]; $t=0.521, \mathrm{ES}=$ $0.19, P=.610$ and $\mathrm{RPE}_{\text {general }}=18.1$ [1.3] vs 18.3 [0.9]; $z=0.926$, $\mathrm{ES}=0.19, P=.334 ;$ Table 3 ).

\section{Discussion}

The principal novel findings of the current study were that acute BJ supplementation increased tension rating prior to, improved performance during, and lowered muscle RPE immediately following a 30-second Wingate test in resistance training participants. These findings are consistent with our experimental hypotheses and

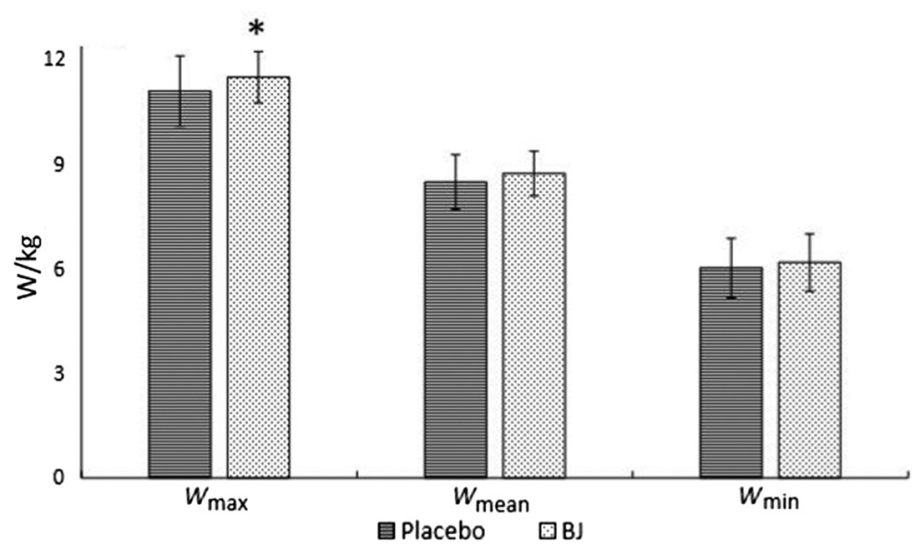

Figure 3 - Power output $(\mathrm{W} / \mathrm{kg}$ ) variables during the Wingate test in the placebo and BJ supplementation conditions. Data are expressed as mean (SD). BJ indicates beetroot juice; $W_{\text {mean }}$, mean power output; $W_{\min }$, minimum power output; $W_{\text {peak }}$, peak power. ${ }^{*}$ Significantly greater than placebo $(P<.05)$. 
Table 2 Scores Reported for the Different Dimensions of the POMS Questionnaire in the 2 Experimental Conditions (Placebo and Beetroot Juice)

\begin{tabular}{lcccc}
\hline Dimension & Placebo & Beetroot juice & ES & P \\
\hline Tension & $3.4(2.4)$ & $4.8(3.0)$ & 0.53 & $.040^{*}$ \\
Depression & $2.3(3.6)$ & $1.4(1.4)$ & 0.34 & .551 \\
Anger & $2.4(3.7)$ & $1.2(2.6)$ & 0.39 & .233 \\
Vigor & $12.5(3.1)$ & $13.3(3.3)$ & 0.26 & .464 \\
Fatigue & $3.7(3.9)$ & $3.1(2.6)$ & 0.19 & .916 \\
Confusion & $13.6(2.4)$ & $13.5(2.4)$ & 0.04 & .926 \\
\hline
\end{tabular}

Abbreviations: ES, effect size; POMS, profile of mood states. Note: Data are expressed as mean (SD).

*Significant difference between the placebo and beetroot juice conditions $(P<.05)$.

Table 3 Scores Reported for RPE in the 2 Experimental Conditions (Placebo and Beetroot Juice)

\begin{tabular}{lcccc}
\hline Dimension & Placebo & Beetroot juice & ES & P \\
\hline $\mathrm{RPE}_{\text {muscular }}$ & $18.3(1.0)$ & $17.7(1.6)$ & 0.47 & $.031^{*}$ \\
$\mathrm{RPE}_{\text {cardio }}$ & $17.7(1.6)$ & $17.4(1.6)$ & 0.19 & .610 \\
$\mathrm{RPE}_{\text {general }}$ & $18.3(0.9)$ & $18.1(1.3)$ & 0.19 & .334 \\
\hline
\end{tabular}

Abbreviations: ES, effect size; RPE, rating of perceived exertion. Note: Data are expressed as mean (SD).

* Significant difference between the placebo and beetroot juice conditions $(P<.05)$.

suggest that improvements in psychophysiological processes might also contribute to the ergogenic effects of $\mathrm{NO}_{3}{ }^{-}$supplementation during short-duration, high-intensity exercise.

In the present study, participants consumed $70 \mathrm{~mL}$ of $\mathrm{BJ}$ or placebo providing $\sim 6.4$ and $0.04 \mathrm{mmol}$ of $\mathrm{NO}_{3}{ }^{-}$, respectively. This dose of $\mathrm{NO}_{3}{ }^{-}$has been reported to increase plasma [nitrite] in numerous studies,,$^{20}$ with the subsequent reduction of circulating nitrite to $\mathrm{NO}$ believed to underpin the ergogenic effects of $\mathrm{NO}_{3}{ }^{-}$ supplementation. ${ }^{2}$ Therefore, although plasma [nitrite] was not assessed in the present study, we adopted a protocol that has been consistently shown to enhance plasma [nitrite] by a magnitude that would be expected to improve exercise performance. Indeed, acute supplementation with a similar dose of $\mathrm{NO}_{3}{ }^{-}$has previously been reported to enhance performance. ${ }^{21}$

The acute ingestion of BJ increased $W_{\text {peak }}$ compared with the placebo condition $(+4.4 \%)$, consistent with some previous studies reporting increased $W_{\text {peak }}(\sim+6 \%)$ in the Wingate test ${ }^{22}$ and other protocols designed to assess $W_{\text {peak }}$ on a cycle ergometer. ${ }^{23}$ In addition to this improvement in $W_{\text {peak }}$, we observed a reduction in the time-to- $W_{\text {peak }}$ following BJ ingestion. This improvement in time-to- $W_{\text {peak }}$ is also in line with a previous observation ${ }^{24}$ following $\mathrm{BJ}$ consumption. As a greater and more rapid attainment of $W_{\text {peak }}$ is an important determinant of performance in team sports and sprint events in athletics, track cycling, or speed skating, our findings might have implications for improving performance in these sports. Indeed, $\mathrm{NO}_{3}{ }^{-}$supplementation has been reported to improve performance during single sprints and repeated bouts of high-intensity exercise in certain experimental conditions..$^{15,24}$

The improvements in $W_{\text {peak }}$ and time-to- $W_{\text {peak }}$ in the present study were accompanied by modifications to some factors that define an individual's mood state, as assessed via the POMS questionnaire. Specifically, compared with placebo, supplementation with BJ increased tension ratings. Although some studies have observed an increased tension rating concomitant with compromised exercise performance following caffeine supplementation, ${ }^{25}$ there is also evidence to suggest that increased tension reflects an optimal state of emotional preparation to undertake a physical task.
Indeed, tension has been suggested to exhibit a parabolic relationship with exercise performance whereby levels of tension that are too low or too high will elicit suboptimal performance. Therefore, our findings of improved $W_{\text {peak }}$ and time-to- $W_{\text {peak }}$ accompanied by increased tension following $\mathrm{BJ}$ supplementation are consistent with the notion that a small elevation in tension can enhance exercise performance. ${ }^{26}$ This potential for increased tension to have enhanced performance in this study might be linked to an increased willingness to commit to the task and/or greater sensations of alertness and optimism when facing the competitive situation.

Several studies have assessed the effect of BJ supplementation on RPE. Although some authors have reported improved performance without any significant impact on RPE, ${ }^{14}$ others have shown performance improvements concomitant with a drop in RPE. ${ }^{15}$ However, a novel contribution of our study was that participants were asked to provide specific RPE scores for $\mathrm{RPE}_{\text {general }}, \mathrm{RPE}_{\text {muscular, }}$, and $\mathrm{RPE}_{\text {cardio }}$ as opposed to just $\mathrm{RPE}_{\text {general, }}$, which has been assessed in previous studies. ${ }^{14,15}$ Our finding that BJ supplementation selectively lowered $\mathrm{RPE}_{\text {muscular, }}$, but not $\mathrm{RPE}_{\text {general }}$ or $\mathrm{RPE}_{\text {cardio, }}$, provides novel information pertaining to the potential mechanism for the ergogenic effect of BJ supplementation.

Among the possible mechanisms that could explain the effects of $\mathrm{BJ}$ on RPE is an enhancement in blood flow to the frontal lobe of the brain. ${ }^{16}$ This brain region processes emotions and decision making and regulates motor control, ${ }^{27}$ which all contribute to the integrative subjective perception of exertion. ${ }^{17} \mathrm{In}$ addition to influencing mood, brain blood flow and oxygenation have important implications for exercise performance. Indeed, reduced blood flow to the brain during an exercise effort has been identified as a factor promoting the onset of fatigue. ${ }^{28}$ Therefore, enhanced brain blood flow could have contributed to the lower $\mathrm{RPE}_{\text {muscular }}$ and improved performance after BJ supplementation in the current study. Alternatively, or in conjunction with enhanced brain perfusion, the lowering in $\mathrm{RPE}_{\text {muscular }}$, but not $\mathrm{RPE}_{\text {general }}$ or $\mathrm{RPE}_{\text {cardio, }}$, after BJ supplementation could be linked to a lower muscle metabolic perturbation ${ }^{4}$ and a subsequent reduction in type III/IV 
skeletal muscle afferent feedback. ${ }^{29}$ Further research is required to resolve the underlying mechanisms for the lower $\mathrm{RPE}_{\text {muscular }}$ after BJ supplementation and its relative importance to the ergogenic effects of this nutritional intervention.

\section{Practical Applications}

In the present study, acute BJ ingestion increased $W_{\text {peak }}$ and lowered the time to achieve this higher $W_{\text {peak }}$, without altering the $W_{\text {mean }}$, compared with the placebo condition. This improvement in variables related to acceleration and peak power output after BJ supplementation might be expected to translate into enhanced acceleration, rate of force development, and peak force development. Subsequently, these enhancements might be expected to improved performance in sports, such as sprinting events in track-and-field athletics, track cycling and speed skating, or powerlifting, where acceleration, rate of force development, and peak force development are key performance determinants. However, further research is required to determine whether the findings from the current study can be reproduced in field settings. It should also be acknowledged that, as subjects were required to abstain from foods rich in $\mathrm{NO}_{3}{ }^{-}$for the duration of this study, this could have contributed to the positive effects of BJ supplementation in the current study.

\section{Conclusions}

The acute ingestion of BJ in resistance-trained athletes improved performance in a 30-second Wingate test, as evidenced by an increased peak power and the shortened time to reach this maximum power. This improvement in Wingate test performance was accompanied by an increased feeling of tension prior to the test and a lower RPE of the leg muscles immediately following the test in the BJ condition compared with the placebo condition. These novel observations suggest that BJ supplementation might have implications for improving performance in speed/power athletes and that this ergogenic effect might be linked, at least in part, to an improvement in psychophysiological processes.

\section{References}

1. Maughan RJ, Burke LM, Dvorak J, et al. IOC consensus statement: dietary supplements and the high-performance athlete. Br J Sports Med. 2018;52(7):439-455. PubMed ID: 29540367 doi:10.1136/ bjsports-2018-099027

. Lundberg JO, Weitzberg E. NO-synthase independent NO generation in mammals. Biochem Biophys Res Commun. 2010;396(1):39-45. PubMed ID: 20494108 doi:10.1016/j.bbrc.2010.02.136

3. Ferguson SK, Holdsworth CT, Wright JL, et al. Microvascular oxygen pressures in muscles comprised of different fiber types: impact of dietary nitrate supplementation. Nitric Oxide. 2015;48:38-43. PubMed ID: 25280991 doi:10.1016/j.niox.2014.09.157

4. Bailey SJ, Fulford J, Vanhatalo A, et al. Dietary nitrate supplementation enhances muscle contractile efficiency during knee-extensor exercise in humans. J Appl Physiol. 2010;109(1):135-148. PubMed ID: 20466802 doi:10.1152/japplphysiol.00046.2010

5. Haider G, Folland JP. Nitrate supplementation enhances the contractile properties of human skeletal muscle. Med Sci Sports Exerc. 2014;46(12):2234-2243. PubMed ID: 24681572 doi:10.1249/MSS. 0000000000000351

6. Tanaka Y, Poole DC, Kano Y. pH homeostasis in contracting and recovering skeletal muscle: integrated function of the microcirculation with the interstitium and intramyocyte milieu. Curr Top Med Chem. 2016;16(24):2656-2663. PubMed ID: 27072709 doi:10.2174/ 1568026616666160413130221

7. Essén B. Glycogen depletion of different fibre types in human skeletal muscle during intermittent and continuous exercise. Acta Physiol Scand. 1978;103(4):446-455. doi:10.1111/j.1748-1716. 1978.tb06239.x

8. Domínguez R, Garnacho-Castaño MV, Cuenca E, et al. Effects of beetroot juice supplementation on a 30-s high-intensity inertial cycle ergometer test. Nutrients. 2017;9(12):1360. doi:10.3390/ nu9121360

9. Marcora SM, Staiano W. The limit to exercise tolerance in humans: mind over muscle? Eur J Appl Physiol. 2010;109(4):763-770. PubMed ID: 20221773 doi:10.1007/s00421-010-1418-6

10. Green P, Suls J. The effects of caffeine on ambulatory blood pressure, heart rate, and mood in coffee drinkers. J Behav Med. 1996;19(2):111-128. PubMed ID: 9132505 doi:10.1007/ BF01857602

11. Stafford LD, Rusted J, Yeomans MR. Caffeine, mood, and performance: a selective review. In: Smith BD, Gupta U, eds. Caffeine and Activation Theory : Effects on Health and Behavior. Boca Raton, FL: Taylor and Francis; 2007:284-310. https://www.taylorfrancis. com/books/9781420006568/chapters/10.1201/9781420006568-20. Accessed January 17, 2019.

12. Mair RG, Onos KD, Hembrook JR. Cognitive activation by central thalamic stimulation: the Yerkes-Dodson law revisited. Dose Response. 2011;9(3):313-331. PubMed ID: 22013395 doi:10.2203/ dose-response.10-017.Mair

13. Pfeiffer KA, Pivarnik JM, Womack CJ, Reeves M, Malina RM. Reliability and validity of the Borg and OMNI RPE scales in adolescent females. Med Sci Sports Exerc. 2002;34(5):S278. https:// insights.ovid.com/medicine-science-sports-exercise/mespex/2002/05/ 001/reliability-validity-borg-omni-rpe-scales/1559/00005768. Accessed January 17, 2019.

14. Shannon OM, Barlow MJ, Duckworth L, et al. Dietary nitrate supplementation enhances short but not longer duration running time-trial performance. Eur J Appl Physiol. 2017;117(4):775-785. PubMed ID: 28251402 doi:10.1007/s00421-017-3580-6

15. Murphy M, Eliot K, Heuertz RM, Weiss E. Whole beetroot consumption acutely improves running performance. J Acad Nutr Diet. 2012;112(4):548-552. PubMed ID: 22709704 doi:10.1016/j.jand. 2011.12.002

16. Presley TD, Morgan AR, Bechtold E, et al. Acute effect of a high nitrate diet on brain perfusion in older adults. Nitric Oxide. 2011;24(1):34-42. PubMed ID: 20951824 doi:10.1016/j.niox.2010. 10.002

17. Williamson JW, McColl R, Mathews D, Mitchell JH, Raven PB, Morgan WP. Hypnotic manipulation of effort sense during dynamic exercise: cardiovascular responses and brain activation. J Appl Physiol. 2001;90(4):1392-1399. PubMed ID: 11247939 doi:10. 1152/jappl.2001.90.4.1392

18. Andersen JL, Aagaard P. Effects of strength training on muscle fiber types and size; consequences for athletes training for high-intensity sport. Scand J Med Sci Sports. 2010;20:32-38. PubMed ID: 20840560 doi:10.1111/j.1600-0838.2010.01196.x

19. Fuentes I, Balaguer I, Meliá JL, García-Merita M. Forma abreviada del Perfil de Estado de Ánimo (POMS). V CongresoNacional de Psicología de La ActividadFísica y El Deporte; 1995.

20. Wylie LJ, Mohr M, Krustrup P, et al. Dietary nitrate supplementation improves team sport-specific intense intermittent exercise performance. Eur J Appl Physiol. 2013;113(7):1673-1684. PubMed ID: 23370859 doi:10.1007/s00421-013-2589-8 
21. Shannon OM, Duckworth L, Barlow MJ, et al. Effects of dietary nitrate supplementation on physiological responses, cognitive function, and exercise performance at moderate and very-high simulated altitude. Front Physiol. 2017;8:401. PubMed ID: 28649204 doi:10. 3389/fphys.2017.00401

22. Cuenca E, Jodra P, Pérez-López A, et al. Effects of beetroot juice supplementation on performance and fatigue in a 30-s all-out sprint exercise: a randomized, double-blind cross-over study. Nutrients. 2018;10(9):1222. doi:10.3390/nu10091222

23. Rimer EG, Peterson LR, Coggan AR, Martin JC. Increase in maximal cycling power with acute dietary nitrate supplementation. Int J Sports Physiol Perform. 2016;11(6):715-720. PubMed ID: 26641379 doi:10.1123/ijspp.2015-0533

24. Jonvik KL, Nyakayiru J, Van Dijk JW, et al. Repeated-sprint performance and plasma responses following beetroot juice supplementation do not differ between recreational, competitive and elite sprint athletes. Eur J Sport Sci. 2018;18(4):524-533. PubMed ID: 29412076 doi:10.1080/17461391.2018.1433722

25. Souissi M, Aloui A, Chtourou H, Aouicha HB, Atheymen R, Sahnoun Z. Caffeine ingestion does not affect afternoon muscle power and fatigue during the Wingate test in elite judo players. Biol Rhythm Res. 2015;46(2):291-298. doi:10.1080/09291016.2014. 994857

26. Stevens MJ, Lane AM, Terry PC. Mood profiling during Olympic qualifying judo competition: a case study testing transactional relationships. J Sports Sci Med. 2006;5(CSSI):143-151. PubMed ID: 24357987.

27. Thomas DD, Liu X, Kantrow SP, Lancaster JR, Jr. The biological lifetime of nitric oxide: implications for the perivascular dynamics of $\mathrm{NO}$ and $\mathrm{O}_{2}$. Proc Natl Acad Sci USA. 2001;98(1):355-360. PubMed ID: 11134509 doi:10.1073/pnas.98.1.355

28. Rooks CR, Thom NJ, McCully KK, Dishman RK. Effects of incremental exercise on cerebral oxygenation measured by near-infrared spectroscopy: a systematic review. Prog Neurobiol. 2010;92(2): 134-150. PubMed ID: 20542078 doi:10.1016/j.pneurobio.2010. 06.002

29. Amann M. Significance of Group III and IV muscle afferents for the endurance exercising human. Clin Exp Pharmacol Physiol. 2012; 39(9):831-835. PubMed ID: 22300329 doi:10.1111/j.1440-1681. 2012.05681.x

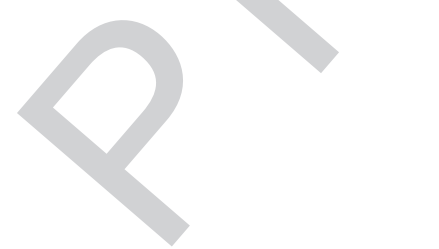

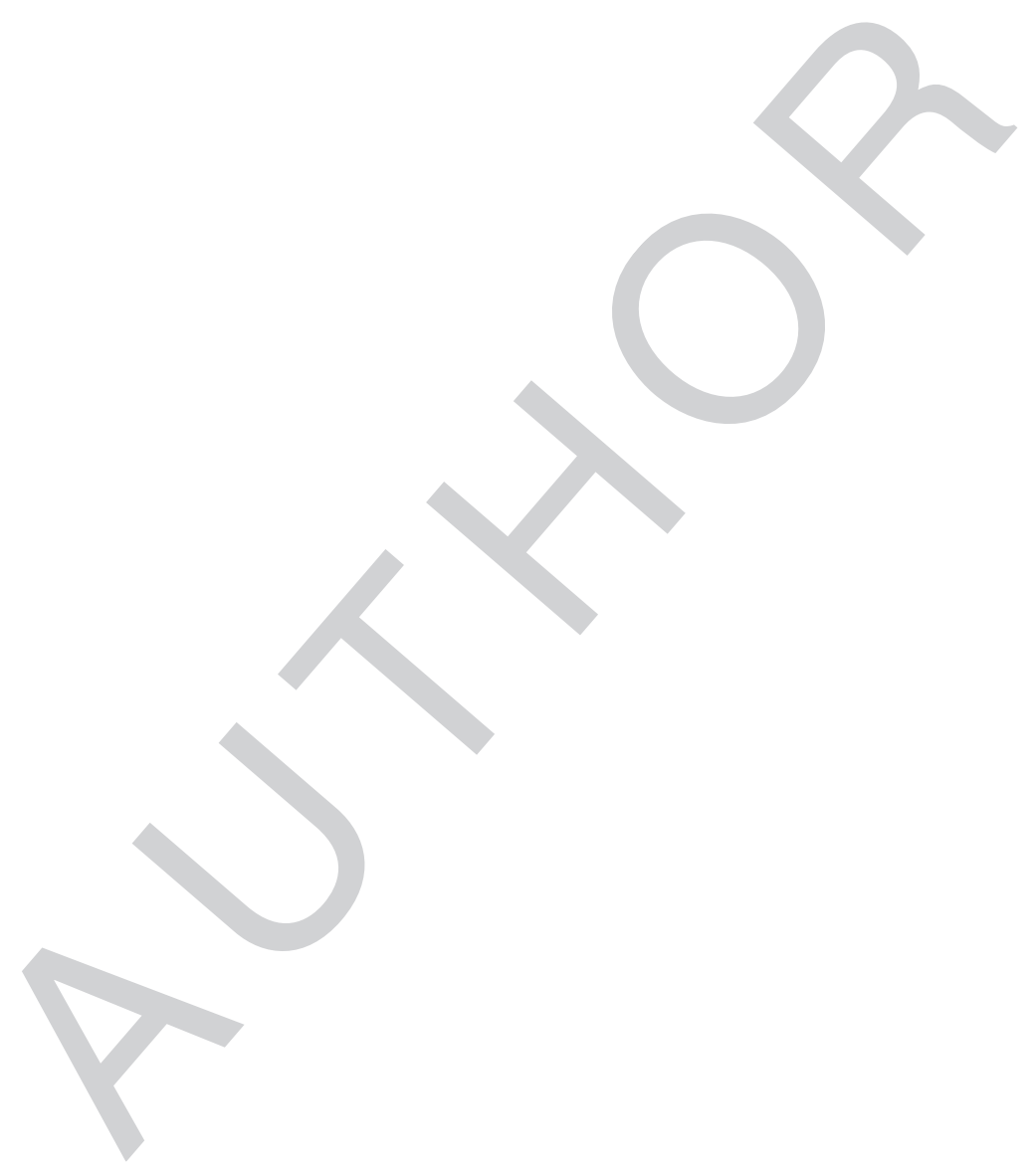


Queries

Q1. Please note that the phrase "which is typically administered in" in sentence starting with "The ergogenic effects" is not grammatically correct in this context (The ergogenic effects are not "typically administered"). Suggest separating for clarity: " $\mathrm{NO}_{3}{ }^{-}$supplementation is typically administered in the form of $\mathrm{NO}_{3}{ }^{-}$-rich beetroot juice (BJ). The erogenic effects of $\mathrm{NO}_{3}{ }^{-}$ supplementation are attributed to....

Q2. Please ensure author information is listed correctly here and within the byline.

Q3. Please check if hr edits to the sentence "The Wingate test was... " are correct and convey the intended meaning

Q4. Please check and confirm the hierarchy of head levels throughout the article.

Q5. As per journal style, "mean \pm SD" should be represented as "mean (SD)." Hence, the values are changed accordingly. Please check and confirm.

Q6. Originally, Refs. 4 and 29 were identical. Hence the duplicate has been removed from the reference list and the subsequent references have been renumbered both in text and in reference list. Please verify.

Q7. Please provide the conference date (ie, day and month) and conference location for Kef 18. 